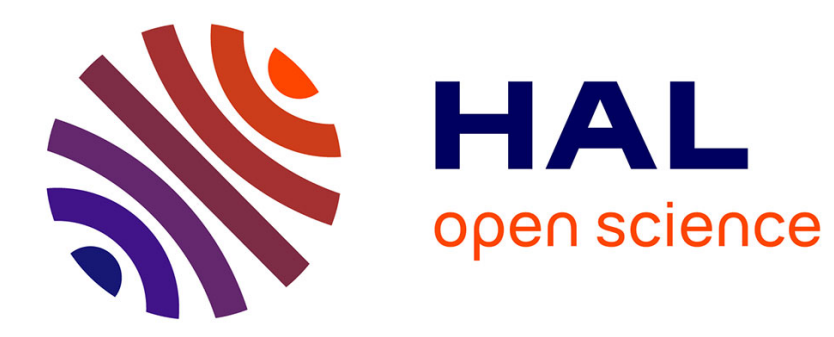

\title{
Recherches sur les diélectriques
}

Julien Lefèvre

\section{To cite this version:}

Julien Lefèvre. Recherches sur les diélectriques. J. Phys. Theor. Appl., 1893, 2 (1), pp.561-563. 10.1051/jphystap:018930020056100 . jpa-00239768

\section{HAL Id: jpa-00239768 https://hal.science/jpa-00239768}

Submitted on 1 Jan 1893

HAL is a multi-disciplinary open access archive for the deposit and dissemination of scientific research documents, whether they are published or not. The documents may come from teaching and research institutions in France or abroad, or from public or private research centers.
L'archive ouverte pluridisciplinaire HAL, est destinée au dépôt et à la diffusion de documents scientifiques de niveau recherche, publiés ou non, émanant des établissements d'enseignement et de recherche français ou étrangers, des laboratoires publics ou privés. 


\title{
REGHERGHES SUR LES DIÉLEGTRIQUES;
}

\author{
Par M. Julien LeFÈVRe.
}

Dans un champ électrique produit par un seul point électrisé $\mathbf{A}$, je place un diélectrique à faces planes et parallèles et je cherche comment la valeur du potentiel se trouve modifiée en un point situé de l'autre côté du diélectrique, sur la perpendiculaire abaissée du point A sur les faces de celui-ci. Je me suis servi d'une balance de Coulomb, carrée et de grandes dimensions, revêtue à l'intérieur d'une couche métallique reliée au sol. Sur la face antérieure est ménagée, pour les observations, une longue fenêtre horizontale, fermée par une glace à faces parallèles. La boule, qui d'ordinaire est fixe, peut décrire une droite horizontale parallèle à cette fenêtre et passant par la position d'équilibre de la boule mobile. Celle-ci est portée par une longue aiguille que soutient un bifilaire, placé excentriquement. Lorsque le bifilaire est sans torsion, cette aiguille est perpendiculaire à la fenêtre; elle porte un petit miroir plan qui permet de le constater par la méthode de Poggendorff. La tête de torsion, qui porte le bifilaire, est placée à l'extrémité d'un fer en T scellé dans un mur épais.

La balance renferme encore un compensateur formé de deux boules ou d'une boule et d'un plateau, disposés de part et d'autre de deux boules principales et destinés à annuler l'action des parois de la cage sur l'aiguille mobile. Les quatre pièces peuvent communiquer avec l'un des pôles d'une bobine de Ruhmkorff, donnant environ ${ }^{\mathrm{cm}}, 5$ d'étincelle et dont l'autre pôle est à la terre.

L'aiguille mobile étant au zéro, c'est-à-dire perpendiculaire à la fenêtre, sans torsion du bifilaire, j'interpose entre les deux boules un diélectrique, solide ou liquide, perpendiculaire à la ligne qui joint leurs centres. Je charge l'aiguille mobile et le compensateur, mais non la boule fixe, et je dispose les deux pièces de celui-ci de façon à maintenir l'aiguille au zéro. Le champ produit sur la boule mobile par les charges des parois, des compensateurs ei de cette boule elle-même, se trouve ainsi annulé.

Je charge alors la boule fixe, que j'amène successivement à dif-

J. de Phys., 3érie, l. II. (Décembre $18 g^{3}$.) 
férentes distances de la boule mobile. Dans chaque position, j'équilibre la répulsion et je ramène l'aiguille au zéro en tordant le bifilaire. La distance des boules est mesurée avec un cathétomètre, placé horizontalement devant la fenêtre.

Je fais ensuite une série d'expériences identiques, mais sans diélectrique. Puis les torsions observées dans ces deux séries subissent deux corrections : la première, relative à l'influence réciproque des deux boules, a été calculée par la méthode des images électriques; elle équivaut à augmenter la distance des centres d'une quantité sensiblement constante, dans les limites de mes expériences; la seconde est relative à la torsion du bifilaire, qui ne suit pas tout à fait la loi du sinus.

Les expériences ainsi corrigées, je construis deux courbes ayant pour abscisses les distances des boules et pour ordonnées les torsions obtenues avec ou sans le diélectrique. Ces courbes montrent que l'effet de la lame isolante est le même, au moins dans les limites de mes expériences, que si l'on rapprochait les boules d'une distance $\delta$, qui paraît être proportionnelle à l'épaisseur $e$ et varier avec la nature de la plaque; je pose donc

$$
\grave{\delta}=e f(k) \text {, }
$$

$k$ étant la constante diélectrique. La distance $\delta$ peut être mesurée directement sur les courbes et j'en déduis $f(k)$, dont les valeurs se trouvent dans le Tableau ci-dessous.

Il reste à déterminer la forme qui convient le mieux pour $f(k)$. J'ai essayé successivement les formes empiriques

$$
\begin{gathered}
\frac{k--\mathrm{I}}{2}, \\
\frac{k-1}{k}, \\
\frac{3}{2} \frac{k-1}{k+2}, \\
2 \frac{k-1}{k+2},
\end{gathered}
$$

qui donnent des résultats identiques pour $k=\mathrm{I}$ et pour $k=2$. Le Tableau suivant montre que les quatre valeurs de $k$, tirées de ces formules, sont suffisamment d'accord avec les nombres de M. Gordon et ceux qui ont été obtenus depuis par divers expéri- 
mentateurs. Il est donc impossible de déterminer avec certitude la forme de $f(k)$.

Parmi les quatre formes étudiées, la premic̀re est celle qui donne, pour une même substance, les nombres les plus concordants; peut-être même peut-on lui reprocher de manquer de sensibilité, car elle fournit, pour les six substances essayées, des valeurs bien voisines les unes des autres. La moyenne pour le soufre est sensiblement inférieure aux nombres de Gordon; de même pour le verre.

La seconde présente au contraire deux défauts de concordance, l'un pour les deux glaces de Saint-Gobain, l'autre pour la plaque de soufre $n^{\circ} 4$, qui ne s'accorde pas avec les trois autres; mais c'est une plaque qui était fondue depuis plus de six mois lorsque je l'ai employée, tandis que les autres ont été introduites dans l'appareil aussitôt après leur fabrication. J'ai d'ailleurs obtenu la même différence dans les mêmes conditions avec d'autres plaques non indiquées ici; mais on sait que, d'après M. Gordon, le pouvoir inducteur du verre augmente notablement avec le temps; il n'est pas surprenant qu'il en soit de même pour le soufre. Le cas des deux glaces de Saint-Gobain peut s'expliquer, soit par la même raison, soit par une différence de composition; rien ne me garantit qu'elles soient formées de verre identique.

\begin{tabular}{|c|c|c|c|c|c|c|c|}
\hline & 0. & & $f(k)$. & $k_{1}$. & $k_{2}$. & $k_{3}$. & $k_{4}$. \\
\hline araffine $b$ & 3,54 & $\mathbf{I}, 775$ & 0,50 & 2,00 & 2,00 & 2,00 & 2,0 \\
\hline$v_{0}$ & 90 & $2, I^{\prime}$ & 0,55 & 2,10 &, 22 & j & , I \\
\hline ite, $n$ & 72 & $\mathrm{I}, 5 \mathrm{o}$ &, 55 & , IO & 22 &, 15 &, $\mathrm{I}^{3}$ \\
\hline 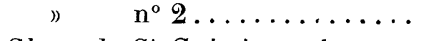 & 12 & $\mathrm{I}, 275$ & $o, 6 o$ & 2,20 & 2,50 & 3 & $2,2\}$ \\
\hline 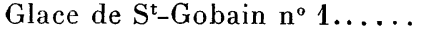 & $\mathbf{1}, 73$ & 20 & $o, 69$ & 2,38 & 3,22 & 70 &, 58 \\
\hline 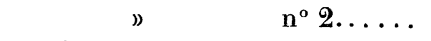 & & I,4 & 0,56 & & 27 & 2,19 & $2, \mathrm{It}$ \\
\hline ufre $n^{\circ} 1$ & 2,87 & 1,825 & $\circ, 63$ & 2,26 & 70 & 2,44 & 2,3 \\
\hline$n^{\circ} 9$ & 4. & 3,00 & o,66 & 2,32 & 4 & 2,57 & 2,4 \\
\hline ” & 3,60 & IO &, 58 & $2, \mathrm{I} 6$ & 2,38 & 2,26 & 2,2 \\
\hline & & & & 2,4 & & 9 & \\
\hline & 3,0 & 1 & 7 & I, & $\mathbf{1}, 58$ & $\mathbf{1}, 6 j$ & 1,6 \\
\hline Ilfu & 3,00 & $\mathrm{I}, 27$ & $0,4^{2}$ & I, 84 & $\mathrm{I}, 72$ & $\mathrm{I}, 77$ & 1,7 \\
\hline
\end{tabular}

